\title{
EXISTENCE AND REPRESENTATION OF SOLUTIONS OF PARABOLIC EQUATIONS ${ }^{1}$
}

\author{
NEIL A. EKLUND
}

ABSTRACT. Let $L$ be a linear, second order parabolic operator in divergence form and let $Q$ be a bounded cylindrical domain in $E^{n+1}$. Let $\partial_{p} Q$ denote the parabolic boundary of $Q$. To each continuous function $f$ on ${ }_{p} Q$ there is a unique solution $u$ of the boundary value problem $L u=0$ in $Q, u=f$ on $\partial_{p} Q$. Moreover, for the given $L$ and $Q$, to each $(x, t) \in Q$ there is a unique nonnegative measure $\mu(x, t)$ with support on $\partial_{p} Q$ such that the solution of the boundary value problem is given by $u(x, t)=\int_{\partial_{p}} Q f^{\prime} d \mu(x, t)^{\circ}$

I. Introduction and preliminary results. Let $\Omega \subset E^{n}$ be a bounded domain with compact boundary, $\partial \Omega$, and let $T>0$. Set $Q=\Omega \times(0, T]$ and let $\partial_{p} Q=\{\partial \Omega \times[0, T]\} \cup\{\Omega \times(0)\}$ denote the parabolic boundary of $Q$. Write $u_{,_{i}}=\partial u / \partial x_{i}$ and $u_{t}=\partial u / \partial t$.

The given functions and solutions will lie in multidimensional $L^{p}$ spaces and the Sobolev space $L^{2}\left[0, T ; H^{1,2}(\Omega)\right]$. These spaces are defined in detail by Aronson and Serrin [2]. The parabolic operator under consideration is defined by

$$
L u=u_{t}-\left\{a_{i j}(x, t) u_{i}+d_{j}(x, t) u\right\},_{j}-b_{j}(x, t) u_{,_{j}}-c(x, t) u
$$

where products involving repeated indices $i$ or $j$ are summed for $1 \leq i, j \leq n$. The results obtained are as follows:

Theorem 1. Let $f \in C\left(\partial_{p} Q\right)$. There is a unique weak solution $u$ of the boundary value problem $L u=0$ in $Q, u=f$ on $\partial_{p} Q$.

Presented to the Society, September 1, 1972 under this title $A$ representation theorem for solutions of parabolic equations with discontinuous coefficients; received by the editors June 19, 1973 and, in revised form, November 7, 1973.

AMS (MOS) subject classifications (1970). Primary 35K20, 35D05; Secondary $35 \mathrm{C} 15$.

Key words and phrases. Parabolic PDE, boundary value problem, existence, integral representation.

${ }^{1}$ Research partially supported by the United States Air Force Office of Scientific Research under contract number AF-AFOSR 883-67 and by the Vanderbilt University Research Council. 
Theorem 2. Let $L$ and $Q$ be given. To each $(x, t) \in Q$ there is a unique nonnegative measure $\mu_{(x, t)}$ on $\partial_{p} Q$ such that the solution $u$ corresponding to the data $f$ found in Theorem 1 is given by $u(x, t)=\int f d \mu_{(x, t)}$.

Theorem 1 is an extension of an existence result obtained by Aronson [1] restated below as Theorem $B$.

The coefficients appearing in the operator $L$ will be assumed to satisfy the following assumptions collectively called $(\mathrm{H})$ :

H.1. The $a_{i j}(x, t)$ are measurable functions in $(x, t)$ with

(a) $\left|a_{i j}(x, t)\right| \leq M<\infty$ almost everywhere in $Q$, and

(b) for some $\lambda>0, a_{i j}(x, t) z_{i} z_{j} \geq \lambda|z|^{2}=\lambda \sum_{i=1}^{n} z_{i}^{2}$ for all $z \in E^{n}$ and almost all $(x, t) \in Q$.

H.2. $c(x, t) \in L^{q}\left[0, T ; L^{p}(\Omega)\right]$ for some pair $p, q$ satisfying

$$
1<p, q \leq \infty, \quad n / 2 p+1 / q<1 .
$$

H.3. $b_{j}(x, t), d_{j}(x, t) \in L^{q}\left[0, T ; L^{p}(\Omega)\right]$ for some pair $p, q$ satisfying

$$
2<p, q \leq \infty, \quad n / 2 p+1 / q<1 / 2 .
$$

For easy reference one basic definition and three basic the orems are stated here without proof.

Definition 1. Let $L$ be as described as above. Assume $G(x, t) \epsilon$ $L^{q}\left[0, T ; L^{p}(\Omega)\right]$ where $p, q$ satisfy $(*)$ and $F_{i}(x, t) \in L^{q}\left[0, T ; L^{p}(\Omega)\right]$ where $p, q$ satisfy $(* *) . u(x, t)$ is called a weak solution of the boundary value problem

$$
\begin{aligned}
& L u=G(x, t)+\left\{F_{i}(x, t)\right\}_{i} \text { in } Q, \\
& u(x, t)=0 \quad \text { on } \mathcal{S}=\Omega \times[0, T], \\
& u(x, t)=u_{0}(x) \quad \text { on } \Omega
\end{aligned}
$$

if

(a) $u \in L^{2}\left[\delta, T ; H_{1 \text { oc }}^{1,2}(\Omega)\right] \cap L^{\infty}\left[\delta, T ; L_{\text {loc }}^{2}(\Omega)\right]$ for each $\delta>0$,

(b) $u_{0}(x) \in L^{2}(\Omega)$,

and if, for each $v(x, t) \in C^{1}(Q) \cap C^{0}(\bar{Q})$ which vanishes in a neighborhood of $\delta$,

$$
\int_{0}^{T} \int_{\Omega}\left[a_{i j} u,{ }_{i} v,_{j}+d_{j} v,_{j} u-b_{j} u,{ }_{j} v-c u v-u v_{t}\right] d x d t
$$

$$
=\int_{0}^{T} \int_{\Omega}\left[G v-F_{i} v,_{i}\right] d x d t+\int_{\Omega} u_{0}(x) v(x, 0) d x,
$$


and

(d) $\lim _{t ! 0} \int_{\mathbf{\Omega}} u(x, t) v(x, t) d x=\int_{\mathbf{\Omega}} u_{0}(x) v(x, 0) d x$.

Aronson and Serrin [2] have shown that every weak solution of (2) in $Q$ has a representative that is continuous in $Q$. Henceforth, $u$ will denote the continuous representative of a given weak solution.

Theorem A (Maximum Principle). Suppose $L$ satisfies $(\mathrm{H})$ and let $u$ be the weak solution of $L u=0$ in $Q$. If $u \in C^{0}(\bar{Q})$ and $m_{1} \leq u \leq m_{2}$ on $\partial_{p} Q$. then

$$
\min \left(m_{1}, 0\right)-\mathcal{C}_{1} \leq u(x, t) \leq \max \left(m_{2}, 0\right)+\mathcal{C}_{2} \text { in } \bar{Q}
$$

where $\mathcal{C}$ depends on $Q$ and the data in $(\mathrm{H})$ and

$$
k_{i}=\left|m_{i}\right|\left(\sum_{j=1}^{n}\left\|d_{j}\right\|_{p, q}+\|c\|_{p, q}\right) \quad \text { for } i=1,2 \text {. }
$$

A proof of this theorem can be found in [2].

Theorem B (Existence). Suppose L satisfies $(\mathrm{H})$ and $u_{0}(x), F_{i}(x, t)$, and $G(x, t)$ are as described in Definition 1. Then there is a unique weak solution $u$ of the boundary value problem (2), (3). Moreover, if $\partial \Omega$ is smooth and $u_{0}(x) \in C_{0}^{0}(\Omega)$, then $u \in C(\bar{Q})$.

A proof of this theorem can be found in [1].

Theorem C (Energy Inequality). Let $u$ be a solution of Lu=0 in $Q$ with initial values $u_{0} \in L^{2}(\Omega)$ and let $\zeta=\zeta(x)$ be a nonnegative smooth function such that $\zeta u \in L^{2}\left[0, T ; H_{0}^{1,2}(\Omega)\right]$. Then there is a positive constant C such that

$$
\|\zeta u\|_{2, \infty}^{2}+\left\|\zeta u_{x}\right\|_{2,2}^{2} \leq \mathcal{C}\left\{\int_{\Omega} \zeta^{2} u_{0}^{2} d x+\left\|\zeta_{x} u\right\|_{2,2}^{2}\right\} .
$$

A proof of this theorem can be found in [2]. Finally, weak solutions of $L u=0$ in $Q$ are locally Hölder continuous with exponent depending on the distance of the points to $\partial_{p} Q$.

\section{Existence theorem.}

Theorem 1. Let $L$ and $Q$ be as described above. Let $f(x, t)$ be continuous on $\mathcal{S}$ and satisfy $f(x, 0) \in L^{2}(\Omega)$. Then there is a unique weak solution $u$ of the boundary value problem 


$$
\begin{array}{ll}
L u=0 & \text { in } Q, \\
u(x, t)=f(x, t) & \text { on } \partial_{p} Q .
\end{array}
$$

Proof. Note that $f$ is continuous on $\mathcal{S}$, a compact set; hence $f$ can be continuously extended to $\bar{Q}$. Let $F(x, t)$ denote this extension. Theorem B can be used to solve the boundary value problem $L u=0$ in $Q, u(x, t)=0$ on $\mathcal{S}, u(x, 0)=f(x, 0)-F(x, 0)$ on $\Omega$. Thus, the theorem will follow if the boundary value problem $L u=0$ in $Q, u(x, t)=F(x, t)$ on $\partial_{p} Q$ can be solved.

For the present assume $\partial \Omega$ is smooth. Approximate $F$ on $\partial_{p} Q$ by polynomials $p^{k}(x, t)$ in the supremum norm so that on $\partial_{p} Q$

$$
m_{1}=\min _{\partial_{p} Q} F \leq p^{k}(x, t) \leq \max _{\partial_{p} Q} F=m_{2} .
$$

Extend the domain of $p^{k}$ to $\bar{Q}$ so that the extension $P^{k}(x, t) \in C^{2}(Q)$. Theorem $\mathrm{B}$ can be applied to solve the boundary value problem $L v^{k}=-L P^{k}$ in $Q$, $v^{k}=0$ on $\partial_{p} Q$.

Define $u^{k}(x, t)=v^{k}(x, t)+P^{k}(x, t)$. Then $u^{k}$ satisfies

$$
\begin{array}{ll}
L u^{k}=0 & \text { in } Q, \\
u^{k}(x, t)=p^{k}(x, t) & \text { on } \partial_{p} Q .
\end{array}
$$

The remainder of the proof consists of showing

(A) The solution $u^{k}$ is independent of the extension $P^{k}$ of $p^{k}$ to $\bar{Q}$.

(B) A subsequence of the $u^{k}$ can be obtained which converges weakly in $L^{2}\left[\delta, T ; H_{1 \text { oc }}^{1,2}(\Omega)\right]$ for each $\delta>0$ to a weak solution of $L u=0$ in $Q$.

(C) A subsequence of that obtained in (B) converges uniformly on all compact subsets of $Q$.

(D) The smoothness assumption on $\partial \Omega$ is removed.

(A) Let $P^{k}$ and $\bar{P}^{k}$ be two extensions of $p^{k}$ to $\bar{Q}$ with $P^{k}, \bar{P}^{k} \in C^{2}(Q)$ and let $u^{k}, \bar{u}^{k}$ denote the corresponding solutions to (5). Then, since $\left[P^{k}(x, t)-\bar{P}^{k}(x, t)\right] \in L^{2}\left[0, T ; H_{0}^{1,2}(\Omega)\right]$ and $\lim _{t ! 0}\left[P^{k}(x, t)-\bar{P}^{k}(x, t)\right]=0$, it follows that $U^{k}(x, t) \equiv u^{k}(x, t)-\bar{u}^{k}(x, t)$ satisfies $L U^{k}=0$ in $Q, U^{k}=0$ on $\partial_{p} Q$. Hence, by Theorem $\mathrm{B}, U^{k} \equiv 0$ on $Q$. Therefore, $P^{k}(x, t)$ may be assumed to be a polynomial.

(B) Since $\partial \Omega$ is smooth and $L u^{k}=0$ in $Q, u^{k} \in C(\bar{Q})$ and, by Theorem $A$,

$$
\bar{m}_{1}=\min (m, 0)+C_{k} \leq u^{k}(x, t) \leq \max \left(m_{2}, 0\right)+C_{k_{2}}=\bar{m}_{2}
$$

on $\bar{Q}$. Define 


$$
\|g\|_{Q} \equiv \sup _{\delta>0}\left\{\delta\left[\left\|g_{x}\right\|_{2,2, Q^{\prime}}^{2}+\|g\|_{2, \infty, Q^{\prime}}^{2}\right]^{1 / 2}\right\}+\sup _{Q}|g|
$$

where $Q^{\prime}=\{x \in \Omega$; dist $(x, \partial \Omega)>\delta\} \times(\delta, T]$. Set $m=\max \left(m_{2},-m_{1}\right) \geq 0$. Then, by Theorem $C,\left\|u^{k}\right\|_{Q} \leq C_{m}$. Hence, on each compact subcylinder $C$ of $Q$

$$
\left\|u_{x}^{k}\right\|_{2,2, C}^{2}+\left\|u^{k}\right\|_{2, \infty, C}^{2} \leq\left[\mathcal{C}_{m} / \operatorname{dist}\left(C, \bigodot_{Q}\right)\right]^{2}
$$

Let $\left\{C^{j}\right\}$ be a sequence of open cylinders with $\bar{C}^{j} C C^{j+1}$ and $C^{j} \uparrow Q$. On $C^{1}$, the weak compactness of $L^{2}\left[H^{1,2}(C)\right]$ and (6) imply there is a subsequence $\left\{u^{1, k}\right\}$ of $\left\{u^{k}\right\}$ which converges weakly in $L^{2}\left[H^{1,2}\left(C^{1}\right)\right]$ to $u$. Having obtained the sequence $\left\{u^{j, k}\right\}$ for $C^{j}$, the weak compactness of $L^{2}\left[H^{1,2}\left(C^{j+1}\right)\right]$ and (6) imply there is a subsequence $\left\{u^{j+1, k}\right\}$ of $\left\{u^{j, k}\right\}$ which converges weakly in $L^{2}\left[H^{1,2}\left(C^{j+1}\right)\right]$. Since $\left\{u^{j+1, k}\right\} \subset\left\{u^{j, k}\right\}$, all of the sequences $\left\{u^{j, k}\right\}$ converge weakly to $u$ in $L^{2}\left[H^{1,2}(C)\right]$ for any compact subcylinder $C$ of $Q$. Set $u^{j}=u^{j, j}$. Then $u^{j}$ converges weakly to $u$ in $L^{2}\left[H^{1,2}(C)\right]$. Hence, $u$ satisfies $L u=0$ weakly in $Q$ and, by Theorem C, $\|u\|_{Q} \leq \mathcal{C}_{m}$.

(C) Since the $u^{j}$ satisfy $L u=0$ in $Q$, they are Hölder continuous on any cylinder $C$ with $\bar{C} \subset Q$. Hence, on each such cylinder, the family $\left\{u^{j}\right\}$ is equicontinuous. Then, by Arzela's theorem, there is a subsequence of $\left\{u^{j}\right\}$ which converges uniformly on $C$. By using the sequence $\left\{C^{j}\right\}$ given in (B) and the diagonalization process again, a subsequence of $\left\{u^{j}\right\}$ is obtained which converges uniformly on any compact subset of $Q$ to $u$. It follows from the uniform convergence of $p^{k}$ to $F$ on $\partial_{p} Q$ that $u$ is the weak solution of the boundary value problem. The uniqueness of $u$ follows from Theorem $B$.

(D) Suppose $\partial \Omega$ is not smooth. Then approximate $\Omega$ by smooth domains $\Omega^{k}$ with $\bar{\Omega}^{k} \subset \Omega^{k+1}, \Omega^{k} \uparrow \Omega$, and the argument in (C) applies to each cylinder $Q^{k}=\Omega^{k} \times(0, T]$. Then the discussion in parts (B) and (C) can be repeated to give the unique weak solution $u$ in $Q$.

II. Representation theorem. In this section the following representation is obtained.

Theorem 2. Let $L$ and $Q$ be as described above. Then, for each $(x, t)$ $\epsilon Q$, there is a unique nonnegative measure $\mu_{(x, t)}$ concentrated on $\partial_{p} Q$ such that, for each continuous function $f$ on $\partial_{p} Q$, the solution $u$ of the boundary value problem (4) is given by

$$
u(x, t)=\int_{\partial_{p} Q} f d \mu_{(x, t)^{\bullet}}
$$


Moreover, for constants $a, A$ such that $0<a \leq \int_{\partial_{p} Q} d \mu_{(x, t)} \leq A$, it follows that the solution $u$ satisfies

$$
\min _{\partial_{p} Q}(a f(x, t), A f(x, t)) \leq u(x, t) \leq \max _{\partial} Q(a f(x, t), A f(x, t)) .
$$

Proof. Define for each $(x, t) \in \bar{Q}$ the functional $\Lambda_{(x, t)}$ on $C\left(\partial_{p} Q\right)$ by

$$
\begin{aligned}
\Lambda_{(x, t)} f & =u(x, t) \text { on } Q, \\
& =f(x, t) \text { on } \partial_{p} Q .
\end{aligned}
$$

$\Lambda_{(x, t)}$ is clearly a positive linear functional and the desired result follows from the Riesz representation theorem.

\section{REFERENCES}

1. D. G. Aronson, Non-negative solutions of linear parabolic equations, Ann. Scuola Norm. Sup. Pisa 22 (1968), no. 4, 607-694.

2. D. G. Aronson and James Serrin, Local behavior of solutions of quasilinear parabolic equations, Arch. Rational Mech. Anal. 25 (1967), 81-122. MR 39 \#5952.

3. Neil Eklund, Boundary behavior of solutions of parabolic equations with discontinuous coefficients, Bull. Amer. Math. Soc. 77 (1971), 788-792. MR 45 \#2311.

4. Avner Friedman, Partial differential equations of parabolic type, PrenticeHall, Englewood Cliffs, N. J., 1964. MR 31 \#6062.

5. O. A. Ladyženskaja and N. N. Ural' ceva, Linear and quasilinear equations of elliptic type, "Nauka", Moscow, 1964; English transl., Academic Press, New York, 1968. MR 35 \#1955; 39 \#5941.

6. Walter Rudin, Real and complex analysis, McGraw-Hill, New York, 1966. MR $35 \# 1420$.

DEPARTMENT OF MATHEMATICS, VANDERBILT UNIVERSITY, NASHVILLE, TENNESSEE 37235

Current address: Department of Mathematics, Centre College, Danville, Kentucky 40422 